\title{
Padrões de deslocamento e procura por forragem de novilhas leiteiras em pastagem de capim-mombaça ${ }^{1}$
}

\section{Ana Luisa Palhano2 ${ }^{2}$ Paulo César de Faccio Carvalho ${ }^{3}$, João Ricardo Dittrich ${ }^{4}$, Aníbal de Moraes $^{5}$, Sila Carneiro da Silva ${ }^{6}$, Alda Lúcia Gomes Monteiro ${ }^{7}$}

\author{
1 Dados parciais da tese de Doutorado da primeira autora. \\ 2 FIES: Rua Tobias de Macedo Jr., 333 e UTP: R. Marcelino Champagnat, 505, Curitiba, PR. \\ 3 UFRGS: Rua Bento Gonçalves, 7712, Porto Alegre, RS. \\ 4 UFPR: Rua dos Funcionários, 1540, Curitiba, PR. \\ 5 UFPR: Rua dos Funcionários, 1540, Curitiba, PR. \\ ${ }^{6}$ ESALQ/USP: Rua Pádua Dias, 111, Piracicaba, SP. \\ 7 UFPR: Rua dos Funcionários, 1540, Curitiba, PR.
}

RESUMO - Para avaliar as estratégias de alimentação de bovinos em pastejo, quatro novilhas da raça Holandês Preto e Branco foram mantidas em uma pastage m de capim-mombaça ( Panicum maximum Jacq.) com cinco alturas de dossel, em um delineamento inteiramente casualizado, com duas repetições, durante os meses de fevereiro a abril de 2002 . Os animais experimentais foram avaliados por meio de testes de pastejo de 45 minutos, durante os quais foram determinados o número de bocados, o número de estações alimentares, o número de passos e o tempo de alimentação utilizando-se cronômetros e contadores. Com o aumento da altura do dossel, o número de estações alimentares diminuiu de forma quadrática ( $\hat{\mathrm{Y}}=16,3199-0,2424 \mathrm{x}+0,000996 \mathrm{x}^{2}, \mathrm{R}^{2}=0,9328, \mathrm{P}=0,0031$ ), como resultado da maior disponibilidade de forragem, que reduziu a procura por melhores sítios de pastejo. O número de passos entre as estações alimentares aumentou ( $\hat{\mathrm{Y}}=0,7247+0,01603 \mathrm{x}, \mathrm{R}^{2}=0,6172, \mathrm{P}=0,0043$ ), ampliando a exploração do ambiente de pastejo. Quando a massa de forragem aumentou, os animais reduziram as distâncias totais percorridas ( $\hat{Y}=513,998287-2,659875 x$, $\left.\mathrm{R}^{2}=0,728076, \mathrm{P}=0,0016\right)$, a taxa média de deslocamento ( $\hat{\mathrm{Y}}=11,567445-0,058396 \mathrm{x}, \mathrm{R}^{2}=0,724633, \mathrm{P}=0,0013$ ) e, conseqüentemente, a intensidade de utilização da área $\left(\hat{Y}=9,533139-0,051351\right.$ x, $\left.R^{2}=0,7708, P=0,0005\right)$, como resultado da elevação da altura do dossel, que garantiu elevado consumo de forragem. Os animais ajustam seus padrões de deslocamento e procura de forragem em resposta à estrutura do dossel forrageiro.

Palavras-chave: comportamento de pastejo, estrutura da pastagem, novilhas holandesas, Panicum maximum

\section{Displacement and forage searching patterns of Holstein heifers in mombaçagrass pasture}

\begin{abstract}
To evaluate grazing strategies of cattle on pastures, four Holstein black and white coating heifers were submitted to five sward heights of mombaçagrass (Panicum maximumJacq.) pasture, in a completely randomized design, with two replications, between February and April, 2002. The experimental animals were evaluated during 45 minutes tests. Number of bites, number of feeding stations, number of steps and feeding time were measured, using chronometers and counters. The number of feeding stations per minute decreased quadratically $\left(\hat{Y}=16.3199-0.2424 x+0.000996 x^{2}, R^{2}=0.9328\right.$, $\mathrm{P}=0.0031$ ), from low to tall sward, as a result of the greater available mass present in the highest sward heights, that reduced the searching for other feeding stations. The number of steps between feeding stations increased $(\hat{\mathrm{Y}}=0.7247+0.01603 \mathrm{x}$, $\left.\mathrm{R}^{2}=0.6172, \mathrm{P}=0.0043\right)$ increasing the exploration of pasture environment. With the increase in available forage dry matter, the animals reduced total number of steps $\left(\hat{\mathrm{Y}}=513.998287-2.659875 \mathrm{x}, \mathrm{R}^{2}=0.728076, \mathrm{P}=0.0016\right)$, steps by minute $\left(\hat{\mathrm{Y}}=11.567445-0.058396 \mathrm{x}, \mathrm{R}^{2}=0.724633, \mathrm{P}=0.0013\right)$ and, consequently, the intensity of area utilization $(\hat{\mathrm{Y}}=9.533139$ $\left.-0.051351 \mathrm{x}, \mathrm{R}^{2}=0.7708, \mathrm{P}=0.0005\right)$, as the greater sward height assured high forage intake. The animals showed adaptive feeding strategies to different pasture structures, in an attempt to improve their ingestive efficiency.
\end{abstract}

Key Words: grazing behavior, Holstein heifers, Panicum maximum, pasture structure

\section{Introdução}

O pastejo é um processo de elevada complexidade, que envolve características do herbívoro e do alimento presente em seu ambiente (Prache et al., 1998). Nesse sentido, habilidades cognitivas dos animais relacionadas à percepção, à discriminação, ao aprendizado e à memória são importantes na adaptação dos animais ao seu ambiente alimentar (Roguet 
et al.,1998) e no desenvolvimento de suas estratégias de pastejo (Gordon \& Lascano,1993). Mesmo em sistemas mais intensivos de produção, em que a oportunidade de utilização dessas estratégias é limitada, os animais escolhem em curto prazo (Griffiths et al., 2003a) o local onde pastejar e onde efetuar um simples bocado.

Considerando a estação alimentar como referencial de avaliação, o comportamento dos animais em pastejo pode ser resumido em tempos de procura e movimentação entre estações alimentares, profundidade, área e taxa de bocados na estação alimentar (Griffiths et al., 2003b) e tempo de permanência nas estações alimentares (Stuth, 1991), sendo fundamental nessa escala de observação o entendimento das possíveis regras para utilização e abandono de determinada estação alimentar (Carvalho, 1997).

O processo de procura por forragem determina a taxa de encontro do animal com o alimento, influenciando, portanto, sua percepção quanto à qualidade e à disponibilidade do alimento no ambiente como um todo (Spalinger et al., 1988; Ungar, 1996), fornecendo subsídios quanto ao número e à qualidade dos bocados potenciais presentes (Carvalho et al., 2000).

As estratégias dos animais, desenvolvidas para melhor monitorar os recursos disponíveis, consistem na avaliação do ambiente e no estabelecimento de referências qualiquantitativas desse ambiente, por meio da visão e constante amostragem dos recursos ao seu redor (Milne, 1991).

Assim, ao escolher determinada estação alimentar, o animal permanece até que o consumo de nutrientes diminua a níveis inferiores à média pré-estabelecida para o ambiente como um todo. A partir daí, o animal passa a se deslocar em busca de novos locais que garantam melhor consumo de nutrientes (Charnov, 1976). Portanto, o tempo de permanência nas estações alimentares depende da riqueza em nutrientes nessa estação (Bailey et al., 1996), da percepção de outros locais com melhores oportunidades de ingestão (Baumont et al., 1998; Prache \& Peyraud, 2001), da freqüência de distribuição de sítios de sub e superutilização da forragem em oferta (Quadros et al., 2003) e da distância até a próxima estação alimentar (O’Reagain \& Schwartz, 1995).

O objetivo neste estudo foi avaliar o efeito da estrutura do dossel de uma pastagem de capim-mombaça sobre a estratégia de pastejo de bovinos e os padrões de deslocamento e procura por forragem.

\section{Material e Métodos}

Os tratamentos consistiram de cinco alturas de dossel (60, $80,100,120$ e $140 \mathrm{~cm}$ ), com duas repetições, em uma pastagem de capim-mombaça (Panicum maximum (L.) Jacq.). Foram realizados dez testes de pastejo no período de 12/02/02 a $14 / 04 / 02$, em piquetes de $540 \mathrm{~m}^{2}$, manejados em crescimento livre após corte de igualação ao final do mês de dezembro de 2001. Os tratamentos foram iniciados, portanto, assim que as alturas propostas (tratamentos) foram atingidas.

Os animais experimentais, quatro novilhas da raça Holandês Preto e Branco (peso médio de $150 \mathrm{~kg}$ ), foram acompanhados por mais três de mesma raça e mesmo porte, visando proporcionar efeito de grupo (Arnold, 1987), durante os testes de pastejo.

Em cada dia de avaliação, foram efetuados os procedimentos de caracterização da estrutura do dossel e os testes de pastejo, durante os quais foram coletados os dados relacionados ao padrão de deslocamento dos animais.

A altura do dossel forrageiro foi medida antes e após os testes de pastejo utilizando-se uma régua graduada denominada sward stick (Barthram, 1985). Para quantificação da massa de forragem e de lâminas foliares, foram coletadas cinco amostras da biomassa vegetal aérea por repetição, utilizando-se de um equipamento denominado estratificador com área basal de $0,5625 \mathrm{~m}^{2}$, segmentado a cada $20 \mathrm{~cm}$ de altura a partir do nível do solo. As amostras foram pesadas, separadas nas frações lâminas foliares, colmos + bainhas e material senescente e, em seguida, foram colocadas em estufa de circulação forçada a $65^{\circ} \mathrm{C}$ até peso constante. A massa de forragem foi estimada a partir da média das cinco amostragens. O mesmo procedimento foi empregado para determinação da massa de colmos + bainhas e de lâminas foliares.

Os resultados obtidos para as massas de forragem, de colmos + bainhas e de lâminas foliares foram utilizados no cálculo de suas respectivas densidades volumétricas, considerando-se a massa de forragem ou de lâminas contida no volume representado pela área basal do estratificador e a altura de cada estrato $(20 \mathrm{~cm})$.

A oferta de forragem estabelecida nos tratamentos foi expressa em $\mathrm{m}^{3} / \mathrm{UA}$ (Duru et al., 2000), com base na altura e na área basal do dossel e, ainda, como porcentagem do peso vivo (kg MS. $100 \mathrm{~kg} \mathrm{PV}^{-1}$ ), utilizando-se os valores obtidos em $\mathrm{m}^{3} / \mathrm{UA}$ e a densidade de forragem.

Para estimativa da taxa de utilização da área, foram marcados aleatoriamente, utilizando-se fio colorido de telefone, 100 perfilhos em cada repetição, distribuídos em cinco transectas. Antes e depois dos testes de pastejo, foi feito o registro da ocorrência de pastejo por meio de medições nas lâminas foliares dos perfilhos marcados. A estimativa da taxa de utilização da área pelos animais (TUA) foi calculada conforme modelo proposto por Wade (1991): 
$\mathrm{TUA}=\mathrm{AP} \times \mathrm{f} \times \mathrm{t}^{-1}$ em que $\mathrm{AP}$ corresponde à área do piquete e ao número de perfilhos pastejados e $\mathrm{t}$ ao período de duração dos testes de pastejo $(\mathrm{t})$, expresso em $\mathrm{m}^{2} \cdot \mathrm{min}^{-1}$.

Com base na metodologia adaptada de Penning \& Hooper (1985), foram conduzidos os testes de pastejo, divididos em duas sessões, cuja seqüência é descrita a seguir: após 6 horas de jejum de sólidos e líquidos, no início da manhã, os animais experimentais foram divididos em duas duplas e encaminhados à área experimental junto com os três acompanhantes, de modo que a primeira dupla (animais A e B) permaneceu amarrada próximo aos piquetes, impossibilitada de consumir água e alimentos. Enquanto isso, a segunda dupla (animais C e D) foi avaliada na primeira sessão de pastejo, com duração de 45 minutos, sendo monitorada por quatro avaliadores que trabalharam em duplas, cada um avaliando um dos dois animais, por meio da contagem do número de estações alimentares escolhidas, do número de passos dados pelos animais durante o período do teste, do número de bocados e do tempo de alimentação utilizando-se contadores e cronômetros. Uma estação alimentar é definida como o semicírculo hipotético disponível em frente ao animal que pode ser alcançado sem que seja necessário mover as patas dianteiras (Ruyle \& Dwyer, 1985). Os passos foram contados utilizando-se como critério a movimentação das patas dianteiras e o tempo de alimentação correspondeu ao período efetivo de captura da forragem (Gibb, 1998), não sendo computados os períodos de deslocamento e procura, pois, quando o animal efetuou deslocamento por período superior a 6 segundos, sem ingestão de forragem, os cronômetros foram desligados.
Ao final dos 45 minutos, as duplas de animais foram trocadas, isto é, os animais A e B foram colocados no piquete para serem avaliados conforme procedimentos da primeira sessão de pastejo e os animais C e D foram mantidos desta vez amarrados próximos ao piquete. Ao final dos 45 minutos, os animais foram encaminhados às áreas adicionais, constituintes de pastagem da mesma espécie forrageira.

O delineamento experimental utilizado foi o inteiramente ao acaso, com cinco tratamentos e duas repetições, totalizando-se dez testes de pastejo, e os dados obtidos foram analisados a partir de regressões polinomiais de $1 \underline{\mathrm{o}} \mathrm{e}$ 20 graus utilizando-se o aplicativo STATGRAPHICS. As comparações de médias foram feitas pelo teste Duncan a $5 \%$ de significância.

\section{Resultados e Discussão}

Na Tabela 1 são apresentadas as características estruturais do capim-mombaça avaliadas nas alturas de dossel.

Considerando os testes de pastejo, verificou-se que as ofertas de forragem foram superiores a $300 \mathrm{~m}^{3} \mathrm{UA}^{-1}$ a partir da altura de $80 \mathrm{~cm}$. Segundo Duru et al. (2000), a amplitude ideal para essa variável, durante o ciclo de pastejo em sistemas rotativos conduzidos adequadamente, seria de 300 a $600 \mathrm{~m}^{3} \mathrm{UA}^{-1}$.

Quando expressa em porcentagem do peso vivo, a oferta de forragem excedeu o recomendado por Hodgson \& Brookes (1999), que apontaram que o desempenho animal seria otimizado com ofertas de 10 a $12 \mathrm{~kg}$ de MS. $100 \mathrm{~kg}$ de $\mathrm{PV}^{-1}$.

Tabela 1 - Alturas efetivas do dossel na entrada e saída dos animais da pastagem, oferta de forragem, densidade volumétrica de forragem, de colmos + bainhas e de lâminas foliares e massas de forragem e de lâminas foliares do capim-mombaça

Table 1 - Effective sward heights at the beginning and at the end of the grazing tests, herbage allowance, herbage, stem plus leaf sheath and leaf blade volumetric density, herbage and leaf mass of mombaçagrass

\begin{tabular}{|c|c|c|c|c|c|c|}
\hline \multirow{3}{*}{$\begin{array}{l}\text { Característica estrutural do dossel } \\
\text { Sward structural characteristics }\end{array}$} & \multirow{2}{*}{\multicolumn{5}{|c|}{$\begin{array}{l}\text { Altura do dossel }(\mathrm{cm}) \\
\text { Sward height }\end{array}$}} & \multirow[t]{3}{*}{$\mathrm{CV}(\%)$} \\
\hline & & & & & & \\
\hline & 60 & 80 & 100 & 120 & 140 & \\
\hline Altura de entrada (1) (Pre-grazing sward height) & $58,9 \mathrm{e}$ & $79,3 \mathrm{~d}$ & $96,5 \mathrm{c}$ & $116,6 b$ & $133,0 \mathrm{a}$ & 2,5 \\
\hline Altura de saída (2) (Post-grazing sward height) & $42,7 \mathrm{~d}$ & $59,6 \mathrm{c}$ & $96,4 b$ & $105,3 \mathrm{ab}$ & $114,1 \mathrm{a}$ & 4,8 \\
\hline Oferta de forragem (3) (Forage allowance) & $237 d$ & $293 c$ & $334 b$ & $410 \mathrm{a}$ & $431 \mathrm{a}$ & 3,4 \\
\hline Oferta de forragem (4) (Forage allowance) & $59,4 b$ & $66,8 \mathrm{ab}$ & $76,6 \mathrm{ab}$ & $89,0 \mathrm{ab}$ & $104,4 \mathrm{a}$ & 14,4 \\
\hline Oferta de lâminas (5) (Leaf blade allowance) & $9,9 \mathrm{c}$ & $21,1 \mathrm{bc}$ & $28,8 b$ & $35,7 \mathrm{ab}$ & $46,5 \mathrm{a}$ & 20,3 \\
\hline Densidade (6) (Density) & $1,26 \mathrm{a}$ & $1,14 \mathrm{a}$ & $1,10 \mathrm{a}$ & $1,09 \mathrm{a}$ & $1,22 \mathrm{a}$ & 15,8 \\
\hline Densidade de colmos (7) (Stem density) & $0,45 \mathrm{a}$ & $0,34 \mathrm{a}$ & $0,33 \mathrm{a}$ & $0,41 \mathrm{a}$ & $0,41 \mathrm{a}$ & 25,2 \\
\hline Densidade de lâminas (8) (Leaf blade density) & $0,21 b$ & $0,36 \mathrm{ab}$ & $0,43 \mathrm{a}$ & $0,44 \mathrm{a}$ & $0,54 \mathrm{a}$ & 19,0 \\
\hline Massa de forragem (9) (Herbage mass) & $7.569 \mathrm{c}$ & $9.130 \mathrm{bc}$ & $11.063 \mathrm{bc}$ & $13.126 \mathrm{ab}$ & $17.247 \mathrm{a}$ & 13,2 \\
\hline Massa de lâminas (10) (Leaf blade mass) & $1.399 \mathrm{~d}$ & $2.911 \mathrm{~cd}$ & $4.114 b c$ & $5.328 b$ & $7.678 \mathrm{a}$ & 17,1 \\
\hline
\end{tabular}

* Médias seguidas da mesma letra, na mesma linha, não diferem $(P<0,05)$ pelo teste Duncan.

* Means followed by the same letter do not differ $(P<0.05)$ by Duncan test.

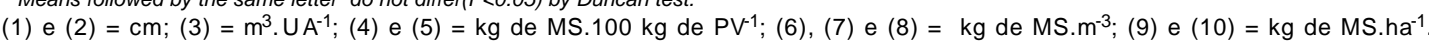

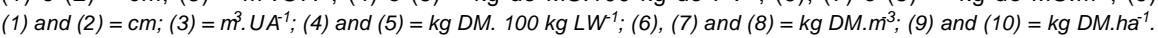


A altura da pastagem não afetou as densidades volumétricas de forragem e de colmos + bainhas do capimmombaça. A estabilização da densidade de lâminas foliares ocorreu a partir da altura de $100 \mathrm{~cm}$. Os resultados encontrados para as densidades volumétricas de forragem e de lâminas foliares se assemelham aos obtidos por Martinichen (2002), que encontrou valores de 0,96 a 1,10 e de 0,39 a 0,48, respectivamente, para o capim-mombaça.

As massas de forragem e de lâminas foliares aumentaram linearmente com a altura do dossel. Martinichen (2002), avaliando duas alturas de pastejo em capim-mombaça, verificou valores de 4.800 a $7.000 \mathrm{~kg}$ de MS de lâminas foliares e de 8.700 a $11.650 \mathrm{~kg}$ de massa de forragem por hectare. No entanto, Uebele (2002), avaliando combinações de intensidade e frequiência de desfolhação em capimmombaça, registrou valores de 4.700 a $8.200 \mathrm{~kg}$ de massa de forragem por hectare, inferiores aos obtidos neste estudo.

Como resposta aos tratamentos, observou-se que, com o aumento em altura do dossel, o número de estações alimentares por minuto foi menor, de 5,6 a 1,5 da menor para a maior altura (Figura 1). Esse comportamento dos animais pode ser explicado pela maior disponibilidade de forragem nas maiores alturas de dossel, condição que, segundo Prache \& Peyraud (2001), pode não motivá-los a trocar de estação alimentar. Segundo Charnov (1976), a redução na disponibilidade de forragem e/ou a percepção de melhores oportunidades de consumo em outros locais favorecem a mudança de estação alimentar.

Paralelamente à redução no número de estações alimentares por minuto, com o aumento na altura do dossel, houve

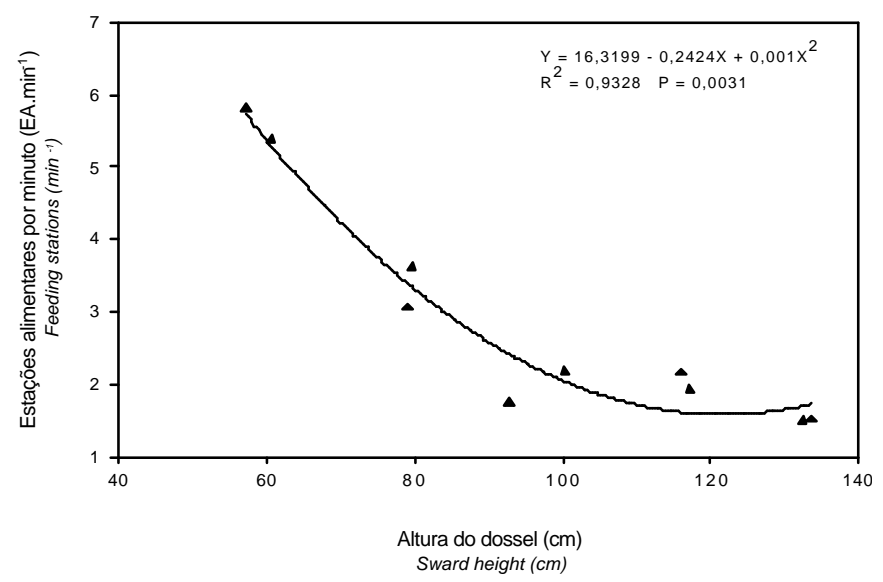

Figura 1 - Relação altura do dossel $(\mathrm{cm}) \times$ número de estações alimentares por minuto visitadas por novilhas holandesas $\left(E A\right.$ min $\left.^{-1}\right)$ em pastagem de capim-mombaça.

Figure 1 - Relationship between sward height $(\mathrm{cm})$ and the number of feeding stations (feeding stations $\mathrm{min}^{-1}$ ) of Holstein heifers grazing mombaçagrass pasture. acréscimo linear no número de passos entre as estações alimentares visitadas (Figura 2), com valores variando de 1,6 a 2,9 passos. Esse comportamento está de acordo com as observações de Prache \& Roguet (1996), segundo as quais, em condições de menor disponibilidade de forragem, os animais caminham menos entre estações alimentares sucessivas, aumentando o número de estações alimentares visitadas, em comparação às situações de ofertas mais elevadas.

O provável aumento da distância percorrida pelos animais entre estações alimentares nas maiores alturas de dossel, caracterizado pelo maior número de passos entre estações, pode ser justificado pelas elevadas massas de bocado em dosséis mais altos, conforme descrito por Carvalho (1997). Ao abandonar uma estação alimentar em direção a outra, é importante para o animal que a troca seja eficiente. Nas maiores alturas, por terem a boca cheia de alimento, os animais podem deslocar-se por mais tempo, antes de eleger uma nova estação alimentar e reiniciar a ingestão de alimento. Isto é provável porque, com alta disponibilidade de forragem, o último bocado de uma estação alimentar ainda apresenta massa elevada e o período correspondente de mastigação permite ao animal deslocar-se por mais tempo enquanto mastiga. A conseqüência é que essa estratégia permite ao animal avaliar melhor o ambiente alimentar disponível, dispensando mais tempo para a procura de melhores sítios de pastejo (Roguet et al., 1998).

No entanto, esse mecanismo é controverso na literatura pois, conforme Trevisan et al. (2003) e Griffiths et al. (2003a), mais que a massa de forragem disponível, a distribuição das

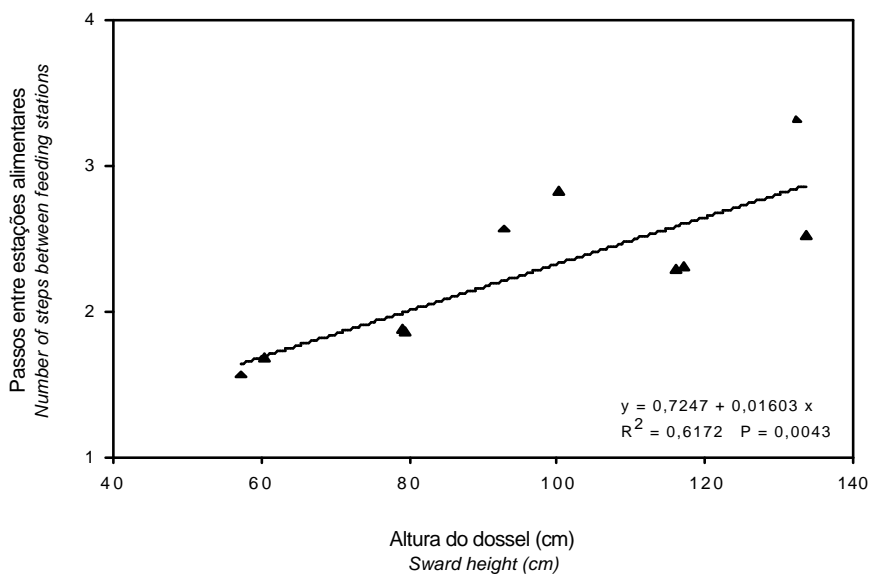

Figura 2 - Relação altura do dossel $(\mathrm{cm}) \times$ número de passos entre estações alimentares de novilhas holandesas em pastagem de capim-mombaça.

Figure 2 - Relationship between sward height $(\mathrm{cm})$ and the number of steps between feeding stations of Holstein heifers grazing mombaçagrass pasture. 
áreas de sub e superpastejo é que seria responsável pela distância entre as estações alimentares eleitas. Segundo esses autores, a estrutura vertical, correspondente ao que foi consumido dentro da estação de "cima para baixo", é que determinaria o tempo de permanência do animal em cada estação alimentar.

Considerando ainda o aproveitamento da estação alimentar pelo animal em pastejo, ao relacionar o número de bocados ao de estações alimentares em diferentes alturas do dossel, obteve-se uma relação quadrática (Figura 3), aumentando a partir das menores alturas de dossel até a altura de $100 \mathrm{~cm}$ e depois diminuindo. A fase ascendente do modelo, de 60 a $100 \mathrm{~cm}$ de altura, está de acordo com a relação teórica entre a riqueza da estação alimentar e sua intensidade de uso (Charnov, 1976). No entanto, a fase descendente, de 120 a $140 \mathrm{~cm}$, comprova a possibilidade de interferência da estrutura do dossel sobre o pastejo. A utilização das estações alimentares tornar-se-ia limitada em condições de dossel com forragem demasiadamente dispersa em seus estratos superiores, em virtude do maior tempo destinado à mastigação e manipulação da forragem (Carvalho et al., 2001; Tharmaraj et al., 2003), o que limitaria a apreensão de novos bocados (Carvalho et al., 2001).

Nas condições avaliadas, o número de bocados por estação alimentar variou de 6 a 10, valores muito próximos ao de 7 bocados por estação alimentar reportados por Wallis de Vries et al. (1998), que trabalharam em pastagens nativas com bovinos.

Apesar do aumento da distância percorrida entre estações alimentares com o incremento na altura do dossel os animais passaram a apresentar menor deslocamento total

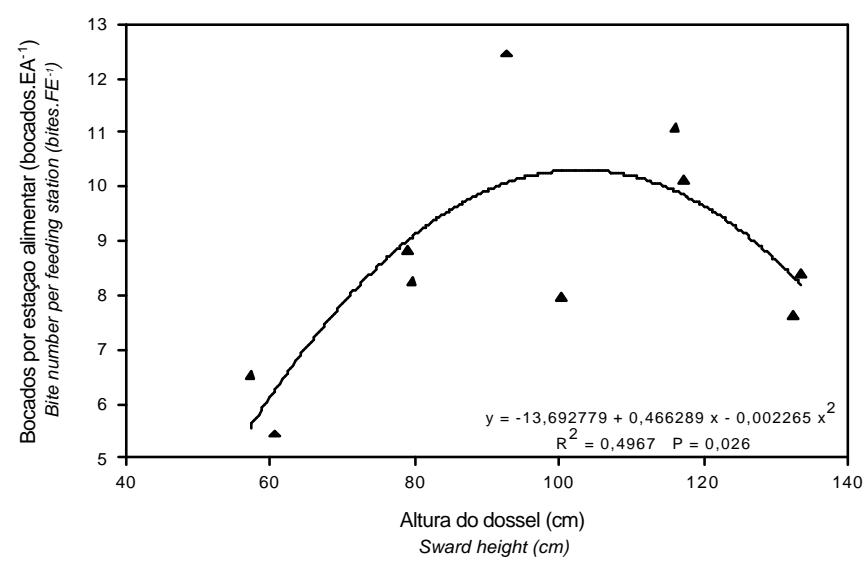

Figura 3 - Relação altura do dossel $(\mathrm{cm}) \times$ número de bocados por estação alimentar (bocados.EA ${ }^{-1}$ ) em capimmombaça.

Figure 3 - Relationship between sward height $(\mathrm{cm})$ and bite number in each feeding station (bites $F S^{-1}$ ) in mombaçagrass pasture. durante os testes de pastejo (Figura 4), bem como velocidades de deslocamento decrescentes (Figura 5), como conseqüência da maior disponibilidade de forragem em pastagens mais altas. Esta situação levou os animais a permanecer mais tempo nas estações alimentares, o que está de acordo com Bailey et al. (1996). Nas menores alturas, portanto, os animais deslocam-se mais e a passos mais velozes. Essa mudança na estratégia de procura da forragem pelo animal, em situação de forragem limitante, provavelmente visa aumentar a taxa de encontro de bocados potenciais na pastagem (Carvalho et al., 1999), com o intuito de manter níveis satisfatórios de consumo.

Como conseqüência da combinação de estratégias de pastejo, houve redução da área utilizada por unidade de tempo com o aumento da altura do dossel (Figura 6), de modo que os valores variaram de 6,24 a 2,70 $\mathrm{m}^{2} /$ animal/ minuto. Considerando a entrada das duas duplas de animais, somados os animais acompanhantes, foram utilizados dez animais, o que gera valores da área utilizada de 0,62 a $0,27 \mathrm{~m}^{2} /$ minuto/animal. Esses resultados são coerentes com aqueles anteriormente verificados, em que os animais caminharam menos e elegeram menor número de estações alimentares, porém explorando-as mais intensivamente à medida que ofereciam maior oferta de alimento (Prache $\&$ Peyraud, 2001). Também Quadros et al. (2003), trabalhando com duas ofertas de lâminas foliares em pastagens de aveia e azevém, observaram menor utilização da área pelos animais na maior oferta de forragem avaliada, o que resultou em elevada heterogeneidade da pastagem.

No outro extremo, o elevado número de passos por minuto, de passos totais e de área explorada por minuto nas

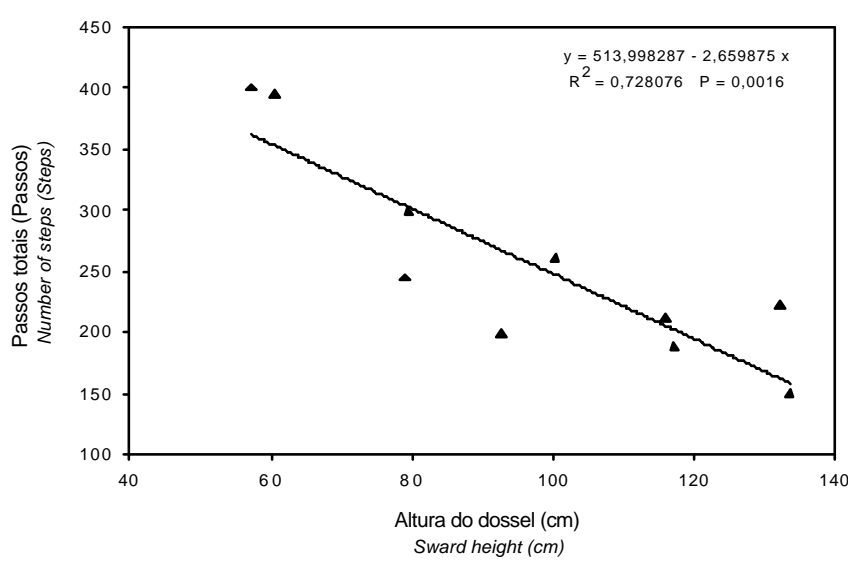

Figura 4 - Relação altura do dossel x número total de passos em pastagem de capim-mombaça.

Figure 4 - Relationship between sward height and total number of steps in mombaçagrass pasture. 


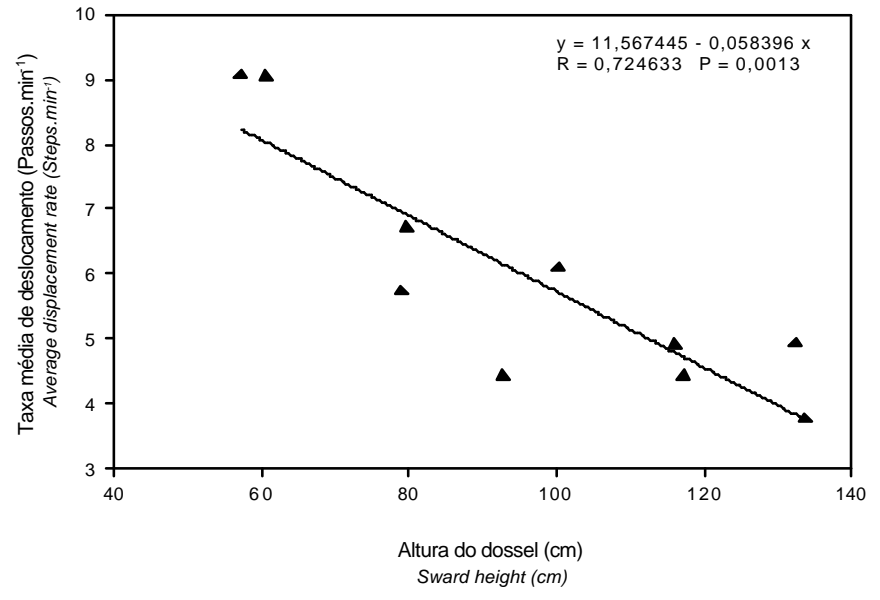

Figura 5 - Relação altura do dossel $(\mathrm{cm}) \times$ taxa média de deslocamento (passos. $\mathrm{min}^{-1}$ ) de novilhas holandesas em pastagem de capim-mombaça.

Figure 5 - Relationship between sward height $(\mathrm{cm})$ and the average displacement rate (steps $\mathrm{min}^{-1}$ ) of Holstein heifers grazing mombaçagrass pasture.

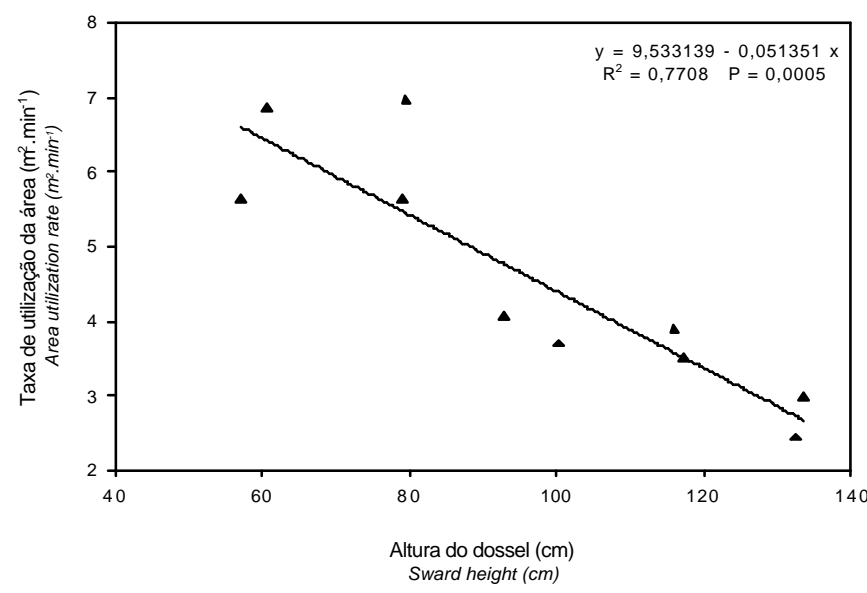

Figura 6 - Relação altura do dossel $(\mathrm{cm}) \times$ taxa de utilização da área $\left(\mathrm{m}^{2} \cdot \mathrm{min}^{-1}\right)$ em pastagem de capim-mombaça.

Figure 6 - Relationship between sward height $(\mathrm{cm})$ and area utilization rate $\left(n^{2} \mathrm{~min}^{-1}\right)$ in mombaçagrass pasture.

menores alturas do dossel, condição também verificada por Prache \& Roguet (1996) com ovinos, promoveu maior mo vimentação durante o processo de busca por forragem, o que pode ser considerado uma tentativa dos animais em adequar seu consumo em situações de massa de forragem restrita (Castro, 2002).

A avaliação conjunta das Figuras 2, 3, 5 e 6 indica que, a partir de $100 \mathrm{~cm}$, o número de estações alimentares foi estabilizado, mas a provável distância entre essas estações continuou aumentando, por meio do incremento do número de passos entre estações alimentares. Se o número total de passos diminuiu com a altura do dossel(Figura 4), mesmo nas condições de pastagens mais altas, os animais passaram a se deslocar com velocidades superiores entre estações alimentares, permanecendo mais tempo em cada es tação. Essa constatação necessitaria de um experimento específico para ser comprovada, pois o tempo de alimentação medido neste trabalho compreendeu conjuntamente os processos de procura (tempo entre duas estações sucessivas) e apreensão (tempo de uso da estação alimentar), sendo necessário medi-los separadamente com vistas à comprovação definitiva. A alteração da velocidade e da direção de deslocamento foi sugerida por Prache et al. (1998) como estratégia dos animais para aumentar as chances de en contrar melhores sítios de pastejo durante as atividades relacionadas à procura por forragem.

\section{Conclusões}

Os padrões de deslocamento e procura de forragem pelos animais são alterados pela estrutura do dossel.

Em situações de menor oferta de forragem, os animais apresentam estratégias alimentares compensatórias, visando aumentar a taxa de ingestão de forragem.

A intensidade de utilização da área diminui com o incremento em altura do dossel, visto que a elevação da massa de forragem disponível nas maiores alturas do dossel não os motiva à procura por outros sítios de pastejo.

\section{Literatura Citada}

ARNOLD, G.W. Grazing behavior. In: SNAYDON, R.W. (Ed.) Managed grassland analytical studies. Oxford: Elsevier, 1987. p.129-135.

BAILEY, D.W.; GROSS, J.E.; LACA, E.A. et al. Mechanisms that result in large herbivore grazing distribution patterns. Journal of Range Management, v.49, p.386-400, 1996.

BAUMONT, R.; PRACHE, S.; MEURET, M. et al. How forage characteristics influence behaviour and intake in small ruminants: a review. In: MEETING ON NUTRITION OF SHEEP AND GOATS, 8., 1998, Grignon. Proceedings... Grignon: 1998. p.2-15.

CARVALHO, P.C.F. A estrutura da pastagem e o comportamento ingestivo de ruminantes em pastejo. In: SIMPÓSIO SOBRE AVALIAÇÃO DE PASTAGENS COM ANIMAIS, 2., 1997, Maringá. Anais... Maringá: Universidade Estadual de Maringá, 1997. p.25-52.

CARVALHO, P.C.F.; PRACHE, S.; DAMASCENO, J.C. O processo de pastejo: desafios da procura e apreensão da forragem pelo herbívoro. In: REUNIÃO ANUAL DA SOCIEDADE BRASILEIRA DE ZOOTECNIA, 36., 1999, Porto Alegre. Anais... Porto Alegre: Universidade Federal do Rio Grande do Sul, 1999. p.253-268.

CARVALHO, P.C.F.; POLI, C.H.E.C.; NABINGER, C. et al. Comportamento ingestivo de bovinos em pastejo e sua relação com a estrutura da pastagem. In: PECUÁRIA 2000: A PECUÁRIA DE CORTE NO III MILÊNIO. 2000, Pirassununga. Anais... Pirassununga: 2000. (CD-ROM). 
CARVALHO, P.C.F.; MARÇAL, G.K.; RIBEIRO FILHO, H.M.N. et al. Pastagens altas podem limitar o consumo dos animais. In: REUNIÃO ANUAL DA SOCIEDADE BRASILEIRA DE ZOOTECNIA, 38., 2001, Piracicaba. Anais... Piracicaba: Sociedade Brasileira de Zootecnia, 2001. p.265-268.

CASTRO, C.R.C. Relações planta-animal em pastagem de milheto (Pennisetum clandestinum (L.) Leeke) manejadas em diferentes alturas com ovinos. Porto Alegre: Universidade Federal do Rio Grande do Sul, 2002. 200p. Dissertação (Mestrado em Zootecnia) - Universidade Federal do Rio Grande do Sul, 2002.

CHARNOV, E.L. Optimal foraging: the marginal value theorem. Theoretical Population Biology, v.9, p.129-136, 1976.

GIBB, M. Animal grazing/intake terminology and definitions. In: PASTURE ECOLOGY AND ANIMAL INTAKE, 3., 1996, Dublin. Proceedings... Dublin: 1998. p.21-37.

GORDON, I.J.; LASCANO, C. Foraging strategies of ruminant livestock on intensively managed grasslands: potential and constraints. In: INTERNATIONAL GRASSLAND CONGRESS, 17., 1993, Palmerston North. Proceedings... Palmerston North: 1993. p.681-690.

GRIFFITHS, W.M. The influence of sward canopy structure on foraging decisions by grazing cattle. I. Patch selection. Grass and Forage Science, v.58, p.112-124, 2003a.

GRIFFITHS, W.M. The influence of sward canopy structure on foraging decisions by grazing cattle. II. Regulation of bite depth. Grass and Forage Science, v.58, p.124-137, 2003b.

HODGSON, J.; BROOKES, I.M. Nutrition of grazing animals. In: WHITE, J.; HODGSON, J. (Eds.) New Zealand pastures and crop sciences. New York: Oxford University, 1999. p.117-132.

MAAK, R. Geografia física do Estado do Paraná. Pinhais: Banco de Desenvolvimento do Paraná, 1968. 350p.

MARTINICHEN, D. Efeito da estrutura do capim-Mombaça sobre a produção de vacas leiteiras. Pinhais: Universidade Federal do Paraná. 2002. 64p. Dissertação (Mestrado em Agronomia) - Universidade Federal do Paraná, 2002.

MILNE, J.A. Diet selection by grazing animals. In: NUTRITION SOCIETY, 50., 1991, Cambridge. Proceedings... Cambridge: CABI Publishing, 1991. p.77-85.

PENNING, P.D.; HOOPER, G.E. An evaluation of the use of shortterm weight changes in grazing sheep for estimating herbage intake. Grass and Forage Science, v.40, p.79-84, 1985.

PRACHE, S.; PEYRAUD, J. Foraging: behavior and intake in temperate cultivated grassland. In: INTERNATIONAL GRASSLAND CONGRESS, 19., 2001, São Pedro. Proceedings... São Pedro: 2001. p.309-319.

PRACHE, S.; GORDON, I.J.; ROOK, A.J. Foraging behaviour and diet selection in domestic herbivores. Annales de Zootechnie, v.48, p.1-11, 1998.

PRACHE, S.; ROGUET, C. Influence de la structure du couvert sur le comportement d'ingestion. Clermont-Ferrand: Institut National de la Recherche Agronomique, 1996. p.22-24. (Rapport d'Activité 1992-1995).
QUADROS, F.L.L.; TREVISAN, N.B.; SILVA, A.C.F. et al. Preferência por sítios de pastejo em pastagem de aveia e azevém submetida a diferentes biomassas de lâmina foliar verde. In: REUNIÃO ANUAL DA SOCIEDADE BRASILEIRA DE ZOOTECNIA, 40., Santa Maria, 2003, Santa Maria. Anais... Santa Maria: Universidade Federal de Santa Maria, 2003. (CD-ROM).

O'REAGAIN, P.J.; SCHWARTZ, J. Dietary selection and foraging strategies of animals on rangeland. Coping with spatial and temporal variability. In: INTERNATIONAL SYMPOSIUM ON THE NUTRITION OF HERBIVORES, 4., 1995, ClermontFerrand. Proceedings... Clermont-Ferrand: 1995. p.419-424.

ROGUET, C.; DUMONT, B.; PRACHE, S. Selection and use of feeding sites and feeding stations by herbivores. A review. Annales de Zootechnie, v.47, p.225-244, 1998.

RUYLE, G.B.; DWYER, D.D. Feeding stations of sheep as an indicator of diminished forage supply. Journal of Animal Science, v.51, n.2, p.349-353, 1985.

SPALINGER, D.E.; HANLEY, T.A.; ROBBINS, C.T. Analysis of the functional response in foraging in the Sitka black-tailed deer. Ecology, v.69, n.4, p.1166-1175, 1988.

STUTH, J. Foraging behavior. In: HEITSCHMIDT, R.K.; STUTH, J. (Ed.) Grazing management: an ecological perspective. [s.1.] 1991, p.85-108.

THARMARAJ, J.; WALES, W.J.; CHAPMAN, D.F. et al. Defoliation pattern, foraging behavior and diet selection by lactating dairy cows in response to sward height and herbage allowance of a rye-grass dominated pasture. Grass and Forage Science, v.98, p.225-238, 2003.

TREVISAN, N.B.; QUADROS, F.L.F.; SILVA, A.C.F. et al. Tempo de permanência entre estações alimentares e distância entre estações de pastejo em pastagem de aveia preta e azevém, submetida a diferentes níveis de biomassa de lâmina foliar verde. In: REUNIÃO ANUAL DA SOCIEDADE BRASILEIRA DE ZOOTECNIA, 40., 2003, Santa Maria. Anais... Santa Maria: Sociedade Brasileira de Zootecnia, 2003. (CD-ROM).

UNGAR, E.D. Ingestive behavior. In: HODGSON, J.; ILLIUS, A. (Eds.) The ecology and management of grazing systems. Wallingford: CABI Publishing, 1996. p.185-218.

WADE, M.H. Factors affecting the availability of vegetative Lollium perenne to grazing dairy cows with special reference to sward characteristics, stocking rate and grazing method. St-Gilles: University of Rennes, 1991. 70p. Thesis (Doctor in Biology Science) - University of Rennes, 1991.

WALLIS DE VRIES, M.F.; LACA, E.A.; DEMMENT, M.W. The importance of scale of patchiness for selectivity in grazing herbivores. Oecologia, v.121, 1999, p.355-363. 